\title{
High-speed and Large Field-of-view Imaging via X-ray Fly-scan Ptychography
}

Yi Jiang ${ }^{1 *}$, Junjing Deng ${ }^{1}$, Jeffrey A. Klug ${ }^{1}$, Yudong Yao ${ }^{1}$, Curt Preissner ${ }^{1}$, Christian Roehrig ${ }^{1}$, Zhonghou Cai ${ }^{1}$, Barry Lai ${ }^{1}$, and Stefan Vogt ${ }^{1}$

1. Advanced Photon Source, Argonne National Laboratory, Lemont, USA

* Corresponding author: yjiang@anl.gov

X-ray ptychography has become a standard tool for high-resolution and non-destructive material characterization, with the state-of-the-art reaching below $10 \mathrm{~nm}$ spatial resolution [1]. In principle, given sufficient overlap, the scan step size is only limited by illumination beam size. Thus, ptychography can be potentially used for obtaining 2D/3D quantitative images of large objects at millimeter and even centimeter-scale. This not only requires new instrumentation and experiment design, but also the development of advanced algorithms for reconstruction and data processing.

The standard X-ray ptychography experiment acquires scanning diffraction patterns in a "move-settlemeasure" fashion. Due to mechanical limitations, a significant amount of time is "wasted" on stage acceleration, deceleration, and stabilization. The problem becomes more prominent with the fourthgeneration synchrotron upgrade, which will increase the coherent flux more than hundred-fold. To reduce the overhead time, a "fly-scan" mode, in which the sample moves continuously during a single exposure, has been developed and demonstrated by several synchrotron groups [2,3,4]. Assuming the observation time is much longer than the coherence time of the incident beam, a fly-scan diffraction pattern can be approximated as an incoherent sum of intensities produced at different measurement times. As a result, traditional ptychographic reconstruction will be blurred along the fly-scan direction. The effect is equivalent to partial coherence and can be corrected by mixed-state ptychography [5], which reconstructs multiple incoherent probe modes using redundant information in the measurement.

At Argonne's Advanced Photon Source, the fly-scan scheme is routinely used for high-throughput ptychography (up to a $3000 \mathrm{~Hz}$ data acquisition rate which is currently limited by detector speed), allowing for large objects imaging and in-situ experiments. As a demonstration, Figure 1 shows ptychographic reconstruction of an integrated circuit with over $550 \times 600 \mu \mathrm{m}^{2}$ field-of-view. The smallest features that can be resolved is about $38 \mathrm{~nm}$. The image is obtained by stitching small regions that are reconstructed independently using the generalized maximum likelihood algorithm [6]. This mitigates the artifacts due to beam variation during long scan time and provides opportunities for parallel computing.

Moreover, the scanning speed can be improved by combining the movement of the sample stage and the probe-generating optics such as a zone plate [7]. The optics stage typically have much less moving mass than the sample stage and provides a faster and more stable scan. However, moving the optics may change the illumination beam on the sample, resulting in a non-uniform phase variation in the reconstruction, as shown in Figure 2a. To account for these discrepancies, we implemented the orthogonal probe relaxation (OPR) method [8] that reconstructs separated probes at each scan position. Combined with mixed-state ptychography, OPR can effectively remove the background in zone-plate scans and improve the reconstruction quality (Figure $2 \mathrm{~b}$ ). The technique is also beneficial to electron ptychography with large probe variation [9]. 
References:

[1] Franz Pfeiffer, Nature Photonics 12 (2018), p.9-17.

[2] J. N. Clark et al., Optical Letters 39 (2014), p.6066-6069.

[3] P. M. Pelz et al., Applied Physics Letters 105, 251101 (2014).

[4] J. Deng et al., Optics Express 23 (2015), p.5438-5451.

[5] P. Thibault and A. Menzel, Nature 494 (2013), p.68-71.

[6] M. Odstrcil et al., Optics Express 26(3) (2018), p.3108-3123.

[7] M. Odstrcil et al., Journal of the Synchrotron Radiation 26(2), (2019).

[8] M. Odstrcil et al., Optics Express 24(8) (2016), p.8360-8369.

[9] This research used resources of the Advanced Photon Source, a U.S. Department of Energy (DOE) Office of Science User Facility operated for the DOE Office of Science by Argonne National Laboratory under Contract No. DE-AC02-06CH11357. Eupholus geoffroyi beetle sample provided by Anatoly Shabalin (University of California San Diego).
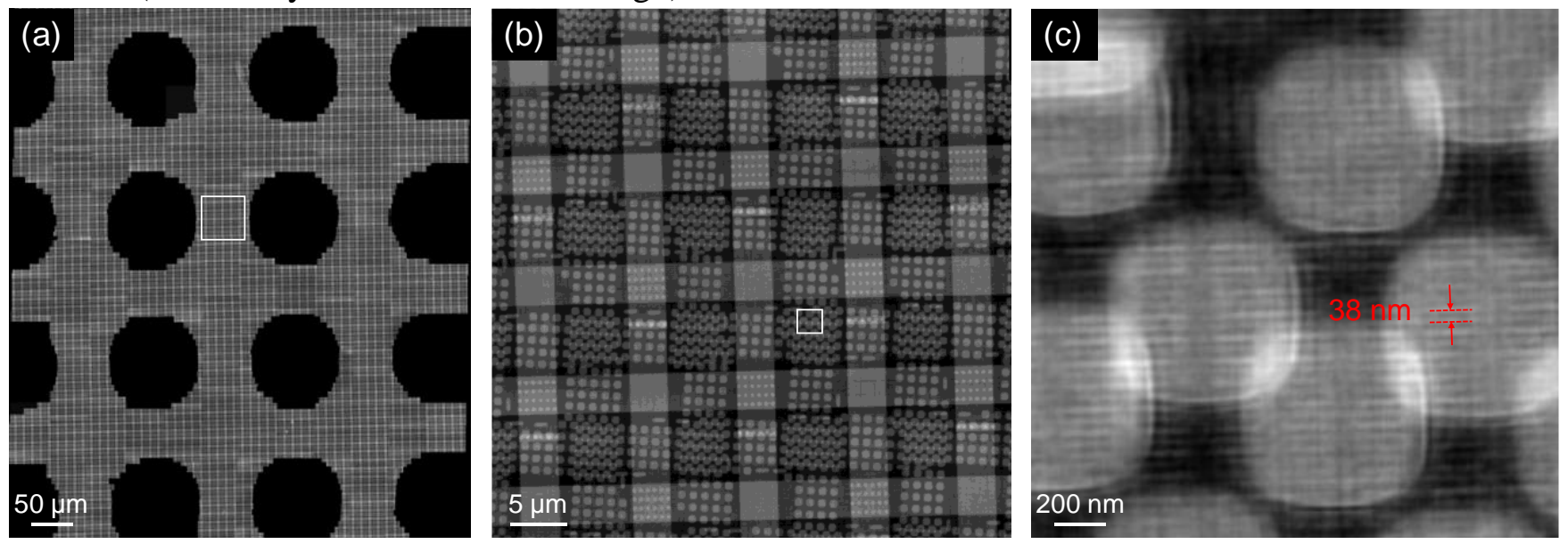

Figure 1. Ptychographic reconstruction of a large integrated circuit. (a) The total field-of-view is over 550 $\times 600 \mu \mathrm{m}^{2}$. The "black holes" are solder bumps that absorb most of X-ray hence were not reconstructed. (b) Close-up of a small region marked in (a). (c) High-resolution reconstruction of the region marked in (b). The pixel size is $6.4 \mathrm{~nm}$ and the smallest features resolved in the reconstruction is $38 \mathrm{~nm}$ apart.

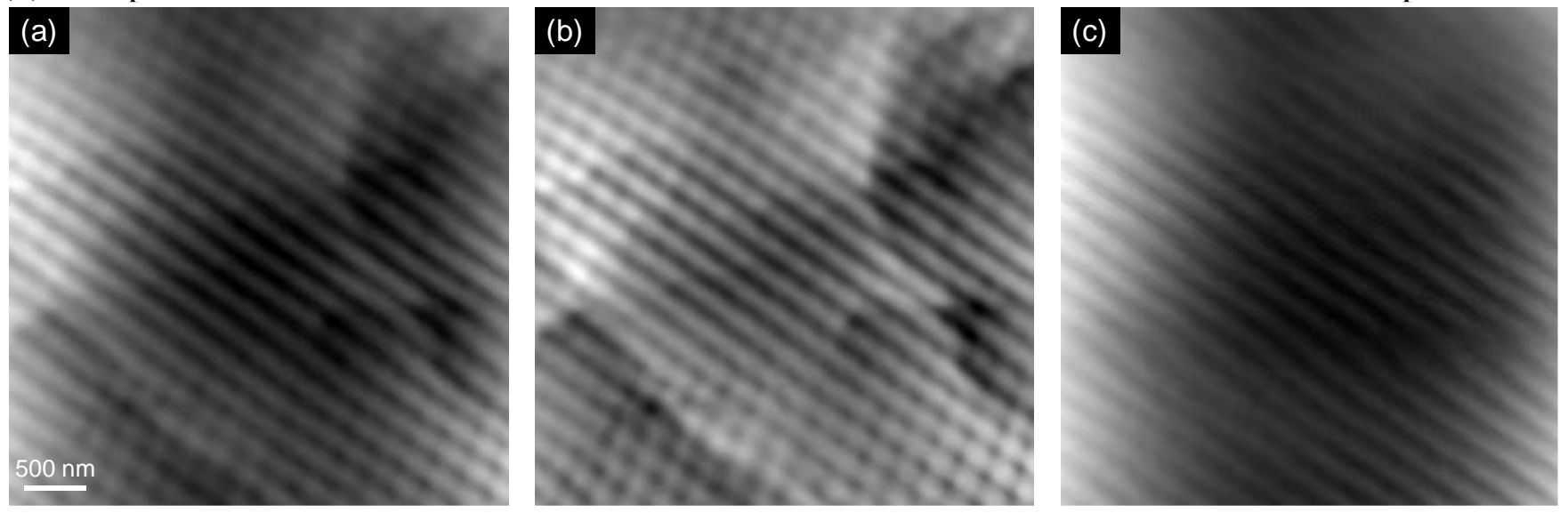

Figure 2. Ptychographic reconstruction of biophotonic crystal structures from Eupholus geoffroyi beetle chitin. The fly-scan data was acquired by moving the zone plate. (a) Reconstructed phase using the mixedstate algorithm. The image has a non-uniform background because the illumination beam changes at each scan position. (b) Reconstructed phase using the mixed-state and orthogonal probe relaxation method, which successfully removes the phase variation. (c) Difference between (a) and (b). 